\title{
Um sistema fuzzy do tipo presa-predador: Aphis glycines x Orius insidiosus
}

\author{
Magda da Silva Peixoto ${ }^{1}$ \\ Depto de Física, Química e Matemática, CCTS, UFSCar \\ 18052-780, Sorocaba, SP \\ E-mail: magda@ufscar.br
}

\author{
Laécio Carvalho de Barros, Rodney Carlos Bassanezi \\ Depto de Matemática Aplicada, IMECC, UNICAMP \\ 13083-859, Distrito de Barão Geraldo, Campinas, SP \\ E-mail: laeciocb@ime.unicam.br, rodney@ime.unicamp.br
}

Resumo: Esse trabalho descreve uma metodologia baseada na Teoria dos Conjuntos Fuzzy e usa um sistema baseado em regras fuzzy para estudar a interação entre a presa, Aphis glycines (pulgão da soja) e seu predador, Orius insidiosus (percevejo). Nosso principal objetivo é a tomada de decisão no controle dessa presa.

\section{Introdução}

O Brasil é hoje o segundo exportador mundial de soja, atrás de EUA e a frente da Argentina. De acordo com o Ministério da Agricultura dos EUA, estima-se que em 2023 o Brasil será o maior exportador de soja do mundo. Segundo as projeções americanas na safra 2022/2023 o Brasil responderá por $44 \%$ das exportações com 63,8 milhões de toneladas seguido dos EUA com 43,8 milhões de toneladas que equivale a 30\% e depois Argentina com 17,5 milhões de toneladas que equivale a 12,1\%. Vale recordar que o Brasil exportou, em 2012, 33,9 milhões de toneladas de soja em grãos, o mesmo de 2011 (disponível em www.fortunaweb.com.ar em 04/03/2013). Considerando a importância econômica da soja para o Brasil, é de fundamental importância que estejamos preparados, com propostas efetivas de controle e combate ao pulgão-da-soja, enquanto essa praga ainda não chegou ao nosso país e, portanto, ainda não causou danos a agricultura nacional.

O pulgão-da-soja, Aphis glycines, introduzido nos Estados Unidos por volta de 2003, tem causado sérios prejuízos à cultura da soja. Diversos inimigos naturais foram observados atacando essa praga. Entretanto, até o momento, foi apenas determinado o limiar econômico de dano para permitir a utilização do controle químico.

Em [6] foi considerado um modelo presa-predador fuzzy para estudar a interação entre pulgões e joaninhas e, em seguida, ajustamos a curva obtida do sistema fuzzy a um modelo clássico do tipo Holling-Tanner, encontrando assim seus parâmetros.

Nessa pesquisa pretendemos elaborar uma metodologia baseada na Teoria dos Conjuntos Fuzzy para estimar a evolução populacional do pulgão-da-soja; elaborar um modelo presapredador por meio de um sistema baseado em regras fuzzy, visando um estudo sobre controle fuzzy da praga via um sistema presa-predador, o qual busca manter a população da praga em um limiar que minimize custos, sem danos ao meio ambiente.

\section{Predação}

A predação é um exemplo da interação entre duas populações que resultam em efeitos negativos no crescimento e na sobrevivência de uma população e positivos ou benéficos para a

\footnotetext{
${ }^{1}$ Bolsista PNPD-CAPES
} 
outra. Predação é o consumo de um organismo (a presa) por outro organismo (o predador). Predadores matam as presas mais ou menos imediatamente após atacá-las [2].

$\mathrm{O}$ modelo de equações diferenciais mais simples é conhecido (como um modelo de competição interespecífica) pelos nomes de seus criadores: Lotka-Volterra. O modelo tem duas componentes: $x$, o número de indivíduos da população de presas e $y$, o número de indivíduos da população de predadores. As hipóteses biológicas do modelo são:

1 - A população de presas aumenta exponencialmente na ausência de predadores;

2 - A diminuição na população de presas depende da taxa de encontros predador-presa;

3 - Estes encontros promovem o aumento no número de predadores;

4 - Na falta de alimento (presa), predadores morrem de fome;

5 - A população de presas aumenta quando a densidade de predadores é baixa e diminui se a densidade for alta;

6 - A população de predadores aumenta quando a densidade de presas é alta e diminui se a densidade for baixa.

Tais hipóteses estão ilustradas na Figura 1 e resumidas nas equações (1):

$$
\left\{\begin{array}{l}
\frac{d x}{d t}=a x-b x y \\
\frac{d y}{d t}=-c y+d x y
\end{array}\right.
$$

sendo,

$a$ : taxa intrínseca de crescimento populacional das presas;

$b$ : eficiência de captura, ou seja, o efeito de um predador sobre o crescimento populacional das presas;

$c$ : a taxa de mortalidade dos predadores na ausência de presas e

$d$ : eficiência de conversão, ou seja, o aumento na população de predadores depende da quantidade de presas devoradas em cada encontro.
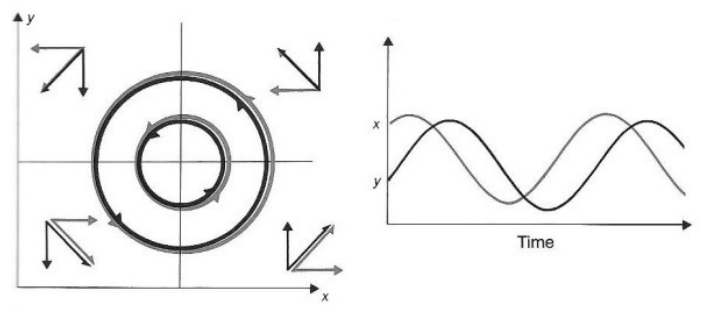

Figura 1: O modelo presa-predador de Lotka-Volterra: plano de fase e contingentes populacionais. Fonte: [2].

Nesse trabalho utilizamos as hipóteses do Modelo de Lotka-Volterra para elaborar o sistema fuzzy do tipo presa-predador.

\section{O modelo matemático}

O pulgão-da-soja, A. glycines, foi introduzido nos EUA por volta do ano de 2003 e tem causados sérios danos a cultura da soja [7]. Estudos têm mostrado que o percevejo Orius insidiosus é um importante predador do pulgão-da-soja [8]. Nessa pesquisa pretendemos elaborar uma metodologia baseada na Teoria dos Conjuntos Fuzzy para elaborar um modelo presa-predador por meio de um sistema baseado em regras fuzzy para estudar a interação entre o pulgão-da-soja, A. glycines e o percevejo Orius insidiosus [3]. Propomos o uso de sistemas baseados em regras fuzzy ao invés de sistemas de equações diferenciais (que caracterizam os modelos clássicos, como o de Lotka-Volterra, por exemplo). Uma vez que temos poucas informações a partir dos dados experimentais, que podem ser relacionadas com o fenómeno, é complicado expressar as variações em função dos estados. Por outro lado, informações 
qualitativas dadas por especialistas permitem propor regras que relacionem (mesmo que parcialmente) as variáveis de estado com suas variações.

Conjuntos Fuzzy e Lógica Fuzzy tornaram-se uma das áreas emergentes no domínio das tecnologias contemporâneas de processamento de informações. A Teoria dos Conjuntos Fuzzy foi desenvolvida por Zadeh [9], em meados da década de 1960 para representar o conhecimento incerto e impreciso. Ele fornece um meio aproximado, mas eficaz, de descrever o comportamento do sistema que é muito complexo, mal definido, com poucos dados. E esse é o nosso caso.

Um subconjunto fuzzy é caracterizado pela função de pertinência que relaciona elementos do domínio $X$ no intervalo [0,1], isto é, $\psi_{A}: X \rightarrow[0,1]$. Então, um conjunto fuzzy $A$ em $X$ pode ser representado como um conjunto de pares ordenados formados por um elemento e seu grau de pertinência: $A=\left\{\left(\psi_{A}(x) / x\right) / x \in X\right\}$. Claramente, um conjunto fuzzy é uma generalização do conceito de um conjunto em que a função de pertinência possui apenas dois valores $\{0,1\}$, isto é, a função característica de $A$.

Variáveis linguísticas (ou fuzzy) são variáveis cujos valores assumidos são conjuntos fuzzy.

Basicamente, sistemas baseados em regras fuzzy (SBRF) possuem quatro componentes: um processador de entrada (fuzzyficação), uma coleção de regras linguisticas, chamada base de regras, um método de inferência fuzzy e um processador de saída (defuzzyficador), gerando números reais como saída. A Figura 2 ilustra a SBRF.

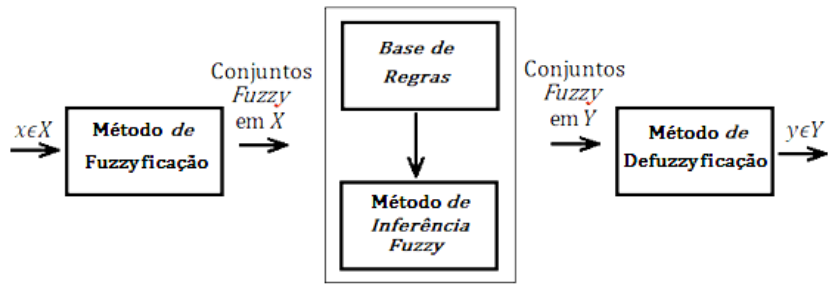

Figura 2.Estrutura de um sistema baseado em regras fuzzy. Fonte: [5]

O método de fuzzyficação corresponde à tradução das entradas do sistema (números reais) por conjuntos fuzzy. Destaca-se a grande importância de especialistas do fenômeno a ser modelado, uma vez que juntamente com eles é que as funções de pertinência são formuladas para cada conjunto fuzzy envolvido no processo.

No nosso modelo, as variáveis do sistema são o número de presas, o número de predadores (entradas), e suas variações (saídas). Os conjuntos fuzzy das variáveis de entrada são qualificados como \{baixa, média baixa, média alta, alta\} e das variáveis de saída são \{alta negativa baixa, baixa negativa, baixa positiva, alta positiva .

A base de regras pode ser considerada como um conjunto de preposições linguísticas do tipo, "Se... então....". Ainda com o auxílio do especialista, as regras estabelecem as relações entre as variáveis linguísticas definidas no sistema.

A partir do plano de fase na Figura 1, ou seja, considerando as hipóteses do modelo de Lotka-Volterra, elaboramos 16 regras do tipo: "Se o número de presas é baixo e o número de predadores é baixo, então a variação de presas é alta positiva e a variação de predadores é alta negativa; Se o número de presas é baixo e o número de predadores á alto, então a variação de presas é alta negativa e a variação de predadores é alta negativa".

É por meio do método de inferência fuzzy que cada proposição fuzzy é "traduzida" matematicamente por meio das técnicas da Lógica Fuzzy. Basicamente, é dele que depende o sucesso do controlador fuzzy, uma vez que ele fornecerá a saída (controle) fuzzy a ser adotada pelo controlador, a partir de cada entrada fuzzy [1]. Nesse trabalho é utilizado o método de inferência fuzzy de Mamdani. Nesse caso, o operador lógico OU é modelado pelo operador máximo que conecta as regras fuzzy da base de regras, e os operadores E e ENTÃO, em cada regra, são modelados pelo operador mínimo 
Finalmente, o método de defuzzyficação consiste em um processo que permite representar um conjunto fuzzy por números reais. Nesse trabalho utilizamos o centróide [4].

\section{Resultados e conclusões}

Nas simulações computacionais observamos a variação do número de presas e do número de predadores ao longo do tempo. Para alcançar esse objetivo, tomamos um número inicial de pulgões, $x_{0}=x\left(t_{0}\right)$, e um número inicial de percevejos, $y_{0}=y\left(t_{0}\right)$, por planta. $\mathrm{O}$ sistema presa-predador fuzzy fornecerá a variações das populações de presa e predador, $x^{\prime}$ e $y^{\prime}$, respectivamente, a partir dos dois valores iniciais dados. Em cada iteração utilizamos o Método de Euler para encontrar $x(t)$ e $y(t)$, ou seja, em cada instante $t$, o número de presas e de predadores são dados pelas fórmulas:

$$
\left\{\begin{array}{l}
x(t)=x\left(t_{0}\right)+\int_{t_{0}}^{t} x^{\prime}(s) d s \\
y(t)=y\left(t_{0}\right)+\int_{t_{0}}^{t} y^{\prime}(s) d s
\end{array}\right.
$$

Consideramos um número inicial de pulgões, $x_{0}=x\left(t_{0}\right)$, e um número inicial de percevejos, $y_{0}=y\left(t_{0}\right)$, por planta (variáveis de entrada do sistema). O sistema fuzzy fornece $x^{\prime}$ e $y^{\prime}$ (variáveis de saída do sistema) e então obtemos $x_{1}=x\left(t_{1}\right)$ e $y_{1}=y\left(t_{1}\right)$ e assim sucessivamente pelo Método de Euler, ou seja,

$$
\left\{\begin{array}{l}
x\left(t_{i+1}\right)=x\left(t_{i}\right)+h x^{\prime}\left(t_{i}\right) \\
y\left(t_{i+1}\right)=y\left(t_{i}\right)+h y^{\prime}\left(t_{i}\right)
\end{array}\right.
$$

onde $\mathrm{h}$ é o incremento.

A Figura 3 apresenta o plano de fase gerado pelo sistema fuzzy (linha continua) e o plano de fase gerado pelos dados reais [3], [4] (linha tracejada).

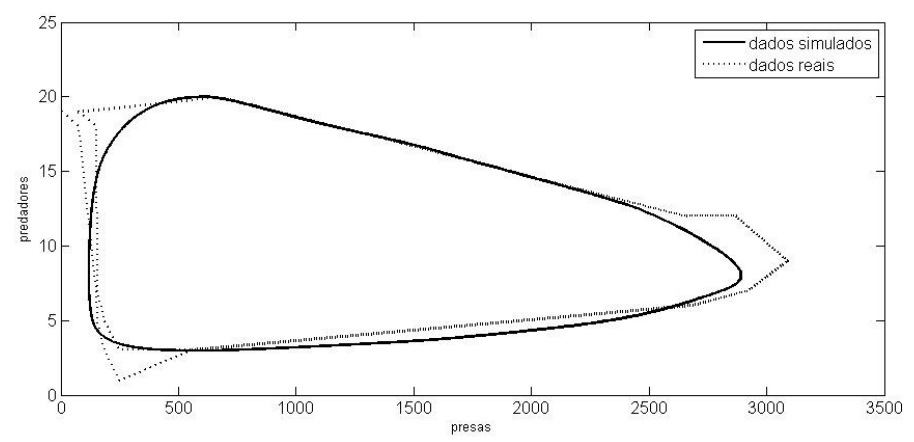

Figura 3. Planos de fase gerados pelo sistema fuzzy e por dados reais com valores iniciais

$$
x_{0}=74 \text { e } y_{0}=3 \text {. }
$$

Dessa forma, o sistema fuzzy fornece a curva solução que preserva as características da curva solução do modelo de Lotka-Volterra.

Nosso estudo sugere que o uso de Teoria dos Conjuntos Fuzzy em Ecologia permite representar a interação entre espécies onde as informações são qualitativas. Nós utilizamos as hipóteses do modelo de Lotka-Volterra e dados fornecidos por especialistas para elaborar um modelo baseado em regras fuzzy sem o uso de equações diferenciais explícitas. 
Consideramos a Teoria dos Conjuntos Fuzzy uma grande contribuição na construção de modelos matemáticos em que nem todos os parâmetros das equações diferenciais são conhecidos ou disponíveis.

Destacamos como vantagens do uso de sistemas baseados em regras fuzzy comparado com modelos determinísticos:

- Os conjuntos das variáveis de entrada e de saída do sistema fuzzy podem ser facilmente definidos por especialistas, ou seja, especialistas definem quando a população de uma determinada espécie é baixa, alta e assim por diante;

- Em um modelo fuzzy, usamos uma base de regras ao invés de sistemas dados por equações, eliminando a dificuldade de se obter parâmetros.

- Havendo necessidade de se conhecer parâmetros, esses podem ser obtidos por meio de um ajuste de curvas da solução gerado pelo modelo baseado em regras fuzzy. Isto é, impõe-se que a curva produzida pelo modelo fuzzy seja uma solução da equação determinística.

\section{Agradecimentos}

A primeira autora agradece à Coordenação de Aperfeiçoamento de Pessoal de Nível Superior (CAPES) pelo auxílio financeiro e o segundo autor ao Conselho Nacional de Desenvolvimento Científico e Tecnológico (CNPq), processo 305862/2013-8.

\section{Referências}

[1] L. C. Barros e R. C. Bassanezi, Introdução à Teoria Fuzzy: Aplicações em Biomatemática, UNICAMP, 2001.

[2] M. Begon, J. L. Harper e C. R. Townsend, Ecology: Individuals, Populations and Communities, Blackwell Scientific Publications, 1990.

[3] T. R. Brosius , L. G. Higley e T. E. Hun, Population Dynamics of Soybean Aphid and Biotic Mortality at the Edge of Its Range, Journal of Economic Entomology 100(4),1268-1275, 2007.

[4] W. Pedrycs e F. Gomide, An Introduction to Fuzzy Sets: Analysis and Design, Massachusets Institute of Technology, 1998.

[5] M. S. Peixoto, Sistemas Dinâmicos e Controladores Fuzzy: um Estudo da Dispersão da Morte Súbita dos Citros em São Paulo; Tese de Doutorado, IMECC/UNICAMP, Campinas/SP, Brasil, 2005.

[6] M. S. Peixoto, L. C. Barros e R. C. Bassanezi, Predator-prey fuzzy model, Ecological Modelling, 214, 39-44, 2008.

[7] D. W. Ragsdale, B. P. McCornack, R. C. Venette, B. D. Potter, I. V. MacRae , E. W. Hodgson , M. E. O’Neal, K. D. Johnson, R. J. O’Neil, C. D. DiFonzo, T. E. Hunt, P. A. Glogoza e E. M. Cullen. Müller and B. D. Serot, Economic Threshold for Soybean Aphid (Hemiptera: Aphididae), Journal of Economic Entomology 110, 1258-1267, 2007.

[8] H. J. S. Yoo e R. J. O'Neil, Temporal relationships between the generalist predator, Orius insidiosus, and its two major prey in soybean, Biological Control 48, 168-180, 2009.

[9] L.A. Zadeh, Fuzzy Sets. Informat. Control, 8, 338-353, 1965. 